\title{
Left Atrial Deformation Predicts Pulmonary Capillary Wedge Pressure in Pediatric Heart Transplant Recipients
}

\author{
Jay Yeh, M.D., ${ }^{*}$ Ranjit Aiyagari, M.D., $\uparrow$ Robert J. Gajarski, M.D., $\uparrow$ Mary C. Zamberlan, C.P.N.P., $\uparrow$ and \\ Jimmy C. Lu, M.D.†
}

*Department of Pediatrics, Division of Pediatric Cardiology, University of California Davis Medical Center, Sacramento, California; and $\dagger$ Department of Pediatrics and Communicable Diseases, Division of Pediatric Cardiology, University of Michigan, Ann Arbor, Michigan

Background: Pulmonary capillary wedge pressure (PCWP) is an important indicator in pediatric heart transplant patients, but commonly used noninvasive surrogates, such as ratio of early diastolic mitral inflow velocity to annular velocity $\left(E / E^{\prime}\right)$, have limitations in this population. This study aimed to evaluate the relation of left atrial (LA) peak systolic strain and distensibility with PCWP in pediatric heart transplant recipients. Methods: Consecutive pediatric heart transplant patients were enrolled at time of cardiac catheterization, with echocardiogram immediately afterward. $E / E^{\prime}$ ratio at the lateral and medial mitral annulus, peak LA systolic longitudinal strain by speckle tracking, and LA distensibility were measured from echocardiograms and compared to invasively measured PCWP. Results: In 38 patients (11.1 \pm 5.8 years old), PCWP correlated with peak LA systolic strain $(r=-0.44, P=0.01)$ and LA distensibility $(r=-0.43, P=0.02)$, but not with $E / E^{\prime}$. On receiver operating characteristics analysis, $L A$ strain had a higher area under the curve than LA distensibility $(0.846$ vs. 0.606$)$. LA strain $<18.9 \%$ had sensitivity $62 \%$ and specificity $95 \%$, with likelihood ratio 12.3 for PCWP $\geq 12$. However, LA strain had lower intra-observer and inter-observer reproducibility than distensibility (intra-class correlation coefficients 0.89 and 0.75 vs. 0.93 and 0.90 ). Conclusions: Peak LA systolic strain and LA distensibility may be more useful surrogates of left ventricular filling pressure than $E / E^{\prime}$ in the pediatric heart transplant population, with greater reproducibility of LA distensibility. Longitudinal studies are needed to evaluate which parameters track changes in PCWP and clinical outcome. (Echocardiography 2015;32:535-540)

Key words: left atrial distensibility, left atrial strain, orthotopic heart transplant, pulmonary capillary wedge pressure

Pediatric heart transplant recipients undergo routine surveillance with invasive measurements of left ventricular (LV) filling pressure. Left ventricular end-diastolic pressure (LVEDP) and its surrogate, pulmonary capillary wedge pressure (PCWP), are elevated in patients with transplant coronary artery disease (TCAD) and rejection., ${ }^{1,2}$ The ratio of peak early mitral inflow velocity (E) to peak early diastolic mitral annular velocity $\left(E^{\prime}\right)$ is commonly used to estimate LVEDP, ${ }^{3-5}$ but is less reliable in patients with $E / E^{\prime}$ of $8-15$ or in patients with preserved systolic function, ${ }^{6,7}$ and may not reliably track changes in PCWP. ${ }^{2,8}$ Left atrial (LA) distensibility ${ }^{9}$ and strain $^{10-13}$ are measures of left atrial reservoir function, and have been shown to reflect left ventricular filling

Address for correspondence and reprint requests: Jimmy $\mathrm{C}$. Lu, M.D., University of Michigan Congenital Heart Center, C.S. Mott Children's Hospital, 1540 E. Hospital Drive, Ann Arbor, Michigan 48109-4204, USA. Fax: (734) 936-9470;

E-mail: jimmyl@med.umich.edu pressure more closely than $E / E^{\prime}$ in various adult populations. However, these measures have not been validated in the pediatric heart transplant population, in whom deformation of the hybrid (donor plus recipient) left atrium has not been evaluated. This study aimed to determine whether LA distensibility or peak strain could better estimate PCWP than $E / E^{\prime}$ in this population.

\section{Methods:}

\section{Study Population:}

In this cross-sectional study, consecutive pediatric heart transplant patients at the University of Michigan Congenital Heart Center were prospectively enrolled from March 2012 to November 2013. Patients were included if they were less than 21 years old, had a history of orthotopic heart transplant, and were undergoing right and/ or left heart catheterization for biopsy or angiography. Patients with mitral stenosis, greater than mild mitral regurgitation, and pulmonary venous 
stenosis were excluded from the study. The institutional review board approved this study and informed consent was obtained from each study patient and/or their guardians.

\section{Echocardiography:}

Echocardiograms were performed using a Vivid E9 cardiovascular ultrasound system (GE Ultrasound, Milwaukee, WI, USA) immediately after catheterization. To minimize risk of sedation, additional sedation medication was not given after the catheterization unless the patient could not tolerate a nonsedated echocardiogram; subjects were typically awake but calm during the echocardiogram. Peak early diastolic mitral inflow velocity $(E)$, peak early diastolic lateral and medial mitral annular tissue Doppler velocities $\left(E^{\prime}\right)$, and velocity of propagation (Vp) were measured per American Society of Echocardiography recommendations. ${ }^{14}$

LA volumes were calculated by biplane area length method from apical four-chamber and two-chamber views (Fig. 1), and were indexed to body surface area. LA distensibility was defined as: (LA maximum volume - minimum volume)/ (LA minimum volume). Maximum and minimum volumes were identified by the frame before mitral valve opening and the frame at mitral valve closure, respectively. Distensibility thus reflects the percentage increase in LA volume during ventricular systole, or the LA reservoir function.

Peak global LA longitudinal systolic strain was also used as a measure of LA reservoir function. Offline analysis was performed from the same apical four-chamber images used for measurement of LA distensibility. Using EchoPAC speckle tracking software (GE Ultrasound), the Automated Function Imaging left ventricular contour was applied to the LA (Fig. 2). The two-chamber view was not used, as it frequently included the LA appendage, and other reports have also used LA strain from a single plane. ${ }^{10}$ Frame rates were greater than 50 frames per second (interquartile range 70-83). Contours were visually evaluated for adequate tracking and adjusted as necessary. Peak global longitudinal strain was manually identified and measured at the highest point of the average strain curve, even if it occurred after aortic valve closure (Fig. 2B). A subset of 30 patients was reanalyzed at least 1 month later by the same experienced observer to evaluate intraobserver variability. To evaluate inter-observer variability, a second experienced observer analyzed the same data, blinded to the other observer's results.

\section{Cardiac Catheterization:}

Cardiac catheterization under conscious sedation was performed on the same day as echocardiog- raphy. Right heart catheterization was performed in all study patients. An end-hole balloon wedge catheter was used to measure right heart chamber pressures and placed in a wedge position to measure mean PCWP. Patients were typically spontaneously breathing, and thus PCWP was measured during exhalation, and averaged over 2-3 respiratory cycles. In patients with a left heart catheterization, a pigtail catheter was advanced retrograde through the aortic valve to measure LVEDP, including simultaneous PCWP and LVEDP measurements. Elevated PCWP was defined as a mean pressure $\geq 12 \mathrm{mmHg}$.

\section{Statistical Analysis:}

Continuous variables are presented as mean \pm standard deviation, if normally distributed, or median with interquartile range. Association of echocardiographic parameters with PCWP measurement was evaluated with Pearson correlation coefficient or Spearman's correlation coefficient
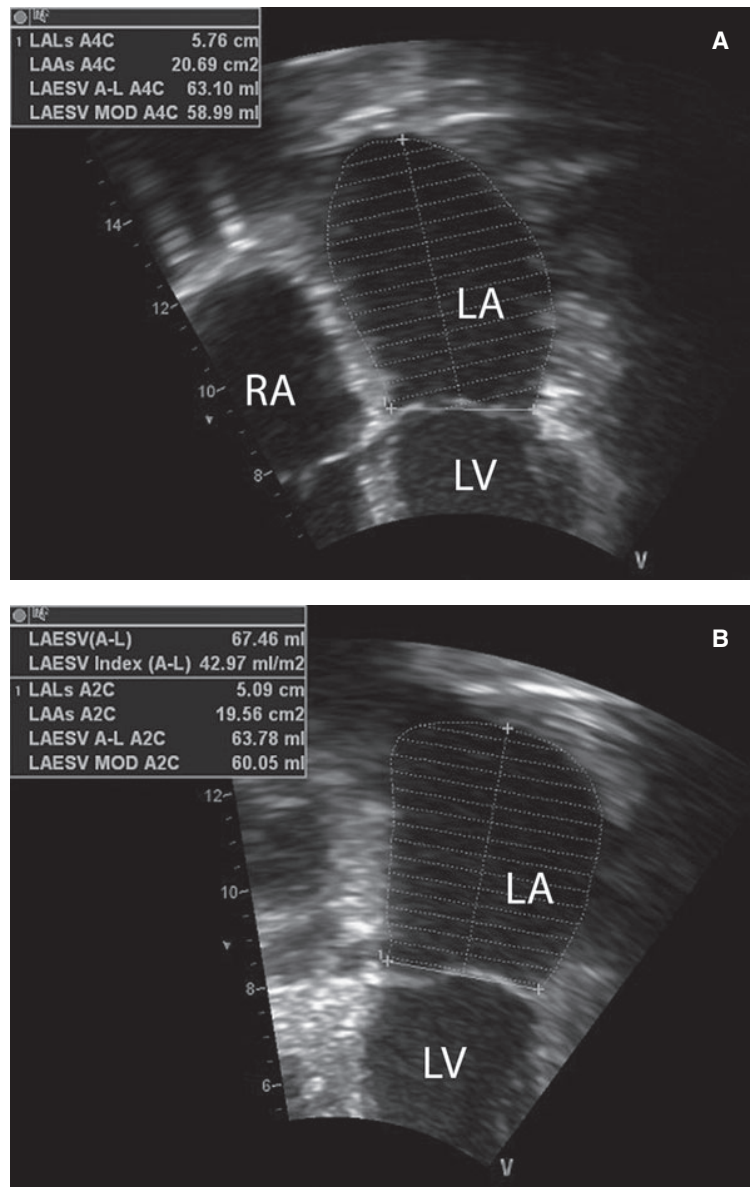

Figure 1. Left atrial volume was measured by biplane area length method from apical A. four-chamber and B. twochamber views. LA, left atrium; LV left ventricle; RA right atrium. 

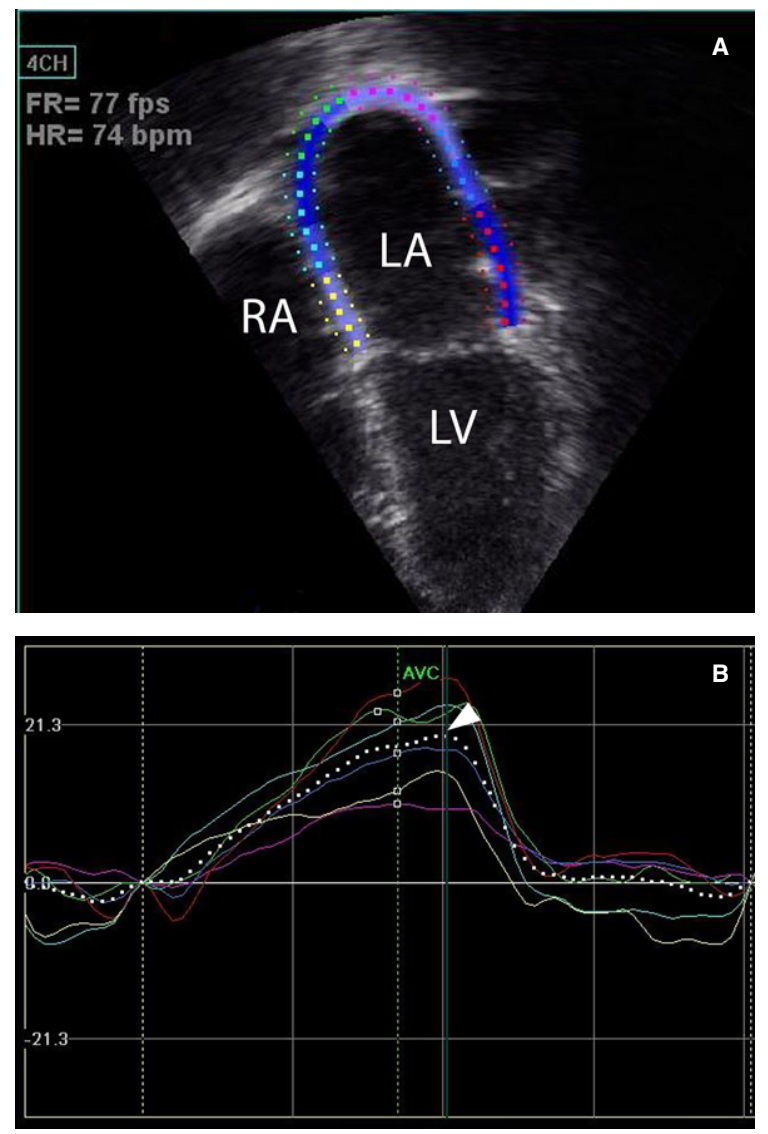

Figure 2. Left atrial peak longitudinal strain was measured from an apical A. four-chamber view. B. The global strain is denoted by the dotted line on the graph. The arrow denotes peak LA longitudinal strain; note that this often occurs after aortic valve closure. LA, left atrium; LV left ventricle; RA right atrium.

for nonparametric variables. Continuous variables were compared with Student's t-test, if normally distributed, or Wilcoxon rank-sum. Receiver operating characteristics (ROC) curve analysis was performed for detection of elevated PCWP. Intraand inter-observer reproducibility were computed by coefficient of variability (COV) and intra-class correlation coefficient (ICC) with 95\% confidence intervals. Two-sided P-values $<0.05$ were considered statistically significant.

\section{Results:}

\section{Patient Characteristics:}

The cohort consists of 38 patients, with demographic characteristics presented in Table I. No patient had more than mild mitral regurgitation, and all patients were in sinus rhythm. Left ventricular ejection fraction was normal in the majority of this cohort, with only three patients below $50 \%$. PCWP was elevated $(\geq 12 \mathrm{mmHg})$ in $14 / 38$

\section{TABLE I}

Patient Demographics, Catheterization, and Echocardiographic Data $(\mathrm{N}=38)$

\begin{tabular}{|c|c|}
\hline Age at study (years) & $11.1 \pm 5.8$ \\
\hline Age at transplant (years) & $5.0(1.8,10.8)$ \\
\hline Female & $16(42 \%)$ \\
\hline Body surface area $\left(\mathrm{m}^{2}\right)$ & $1.15(0.77,1.58)$ \\
\hline \multicolumn{2}{|l|}{ Surgical technique } \\
\hline Biatrial anastomosis & $30(79 \%)$ \\
\hline Bicaval anastomosis & $6(16 \%)$ \\
\hline Unspecified & $2(5 \%)$ \\
\hline $\begin{array}{l}\text { Pulmonary capillary wedge } \\
\text { pressure }(\mathrm{mmHg})\end{array}$ & $9.9 \pm 3.2$ \\
\hline $\begin{array}{l}\text { Left ventricular end-diastolic } \\
\text { pressure }(\mathrm{mmHg})(\mathrm{N}=19)\end{array}$ & $10.1 \pm 2.8$ \\
\hline $\begin{array}{l}\text { Left ventricular ejection fraction } \\
\text { by echocardiogram (\%) }\end{array}$ & $59.8 \pm 7.3$ \\
\hline E/E' ratio, lateral annulus & $7.1(5.9,8.9)$ \\
\hline E/E' ratio, medial annulus & $9.3(8.5,14.7)$ \\
\hline Velocity of propagation $(\mathrm{cm} / \mathrm{s})$ & $62.9(45.0,97.9)$ \\
\hline E/Velocity of propagation & $1.50 \pm 0.58$ \\
\hline $\begin{array}{l}\text { Maximum left atrial volume, } \\
\text { indexed }\left(\mathrm{mL} / \mathrm{m}^{2}\right)\end{array}$ & $24.8(22.3,33.4)$ \\
\hline $\begin{array}{l}\text { Minimum left atrial volume, } \\
\text { indexed }\left(\mathrm{mL} / \mathrm{m}^{2}\right)\end{array}$ & $14.9(11.4,20.5)$ \\
\hline Left atrial distensibility (\%) & $75.3(60.6,97.1)$ \\
\hline Peak left atrial strain (\%) & $21.8(18.2,25.8)$ \\
\hline
\end{tabular}

Data are presented as median (interquartile range), mean \pm standard deviation, or number (percent).

$\mathrm{E}=$ peak early mitral inflow velocity; $\mathrm{E}^{\prime}=$ peak early mitral annular velocity.

patients (37\%), with maximum PCWP $18 \mathrm{mmHg}$. In 19 patients who underwent left heart catheterization, PCWP correlated closely with $\operatorname{LVEDP}(r=0.85, \mathrm{P}<0.0001)$.

On biopsy, three patients had evidence of rejection (International Society of Heart and Lung Transplantation grade 2 or higher). Coronary vasculopathy was present in 2 of 19 patients with coronary angiography; these patients did not have evidence of rejection. One patient had mild paucity of septal perforator branches from the anterior descending and posterior descending arteries. The other patient had $90 \%$ occlusion of the mid-left anterior descending artery, paucity of septal perforators, $40-50 \%$ occlusion of the proximal circumflex, and likely chronic total occlusion of the mid-right coronary.

\section{Predictors of PCWP:}

$E / E^{\prime}$, measured at either the medial or lateral mitral annulus, did not correlate with PCWP (Table II). Among patients with normal versus elevated PCWP, there was no difference in $E / E^{\prime}$ at the lateral annulus (median 7.3 vs. 6.8, $\mathrm{P}=0.91$ ) or medial annulus (median 9.3 vs. 9.4, $\mathrm{P}=0.78$ ). 
TABLE II

Correlation of Echo Parameters with Pulmonary Capillary Wedge Pressure $(\mathrm{N}=38)$

\begin{tabular}{lcc}
\hline Parameter & r-value & P-value \\
\hline E/E' ratio, lateral annulus & 0.25 & 0.17 \\
E/E' ratio, medial annulus & 0.10 & 0.56 \\
Velocity of propagation (Vp) & 0.12 & 0.51 \\
E/Vp ratio & -0.006 & 0.97 \\
Maximum left atrial volume, indexed & 0.24 & 0.22 \\
Minimum left atrial volume, indexed & 0.34 & 0.08 \\
Left atrial distensibility & $-\mathbf{0 . 4 3}$ & $\mathbf{0 . 0 2}$ \\
Peak left atrial longitudinal strain & $\mathbf{- 0 . 4 4}$ & $\mathbf{0 . 0 1}$
\end{tabular}

$\mathrm{E}=$ peak early mitral inflow velocity; $\mathrm{E}^{\prime}=$ peak early mitral annular velocity. Statistically significant values are presented in bold print.

Maximum and minimum left atrial volumes did not correlate with PCWP, but LA distensibility had a modest inverse correlation with PCWP (Table II). Patients with LA distensibility below $74.2 \%$ had a higher PCWP than those with greater distensibility (median $12.0 \mathrm{mmHg}$ vs. $8.0 \mathrm{mmHg}, \mathrm{P}=0.002$, Fig. 3). However, on ROC analysis (Fig. 4), area under the curve was low (0.606). LA distensibility $<74.2 \%$ had a sensitivity of $70 \%$ and specificity of $67 \%$ for elevated PCWP, with likelihood ratio 2.1. LA distensibility correlated with left ventricular ejection fraction $(r=0.48, P=0.01)$.

LA strain could not be evaluated in 4 patients due to inadequate tracking of atrial segments. Peak LA systolic longitudinal strain had a modest inverse correlation with PCWP (Table II). Patients with elevated PCWP had a lower LA strain (median $17.9 \%$ vs. $23.6 \%, P=0.0005)$. Patients with LA strain $<18.9 \%$ had a higher PCWP (median

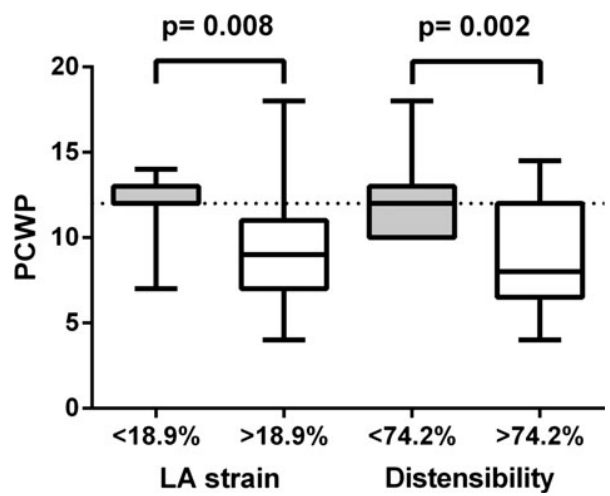

Figure 3. Box and whisker plot for pulmonary capillary wedge pressure (PCWP) versus left atrial strain or distensibility. Box plot denotes median and interquartile range, with whiskers denoting maximum and minimum. Dotted line denotes cutoff for elevated PCWP of $12 \mathrm{mmHg}$.
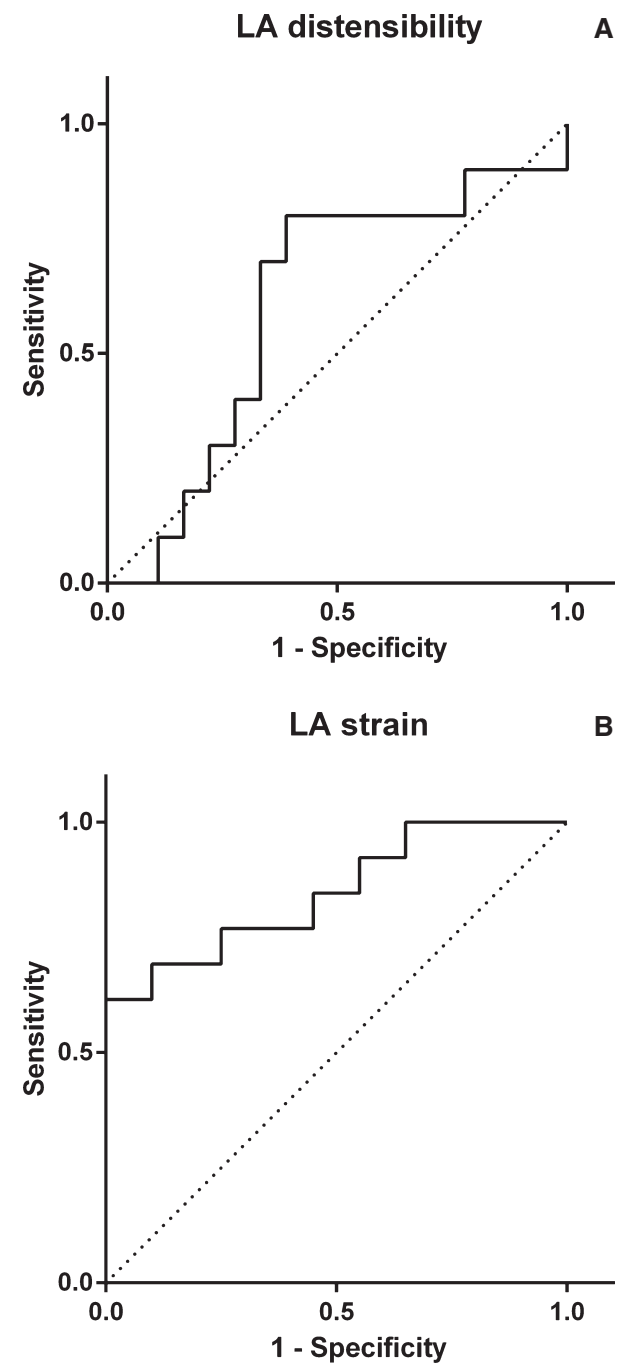

Figure 4. Receiver operating characteristics curve analysis for detection of pulmonary capillary wedge pressure $\geq 12 \mathrm{mmHg}$. A. Left atrial distensibility. B. Left atrial peak systolic strain.

$12.0 \mathrm{mmHg}$ vs. $9.0 \mathrm{mmHg}, \mathrm{P}=0.008$, Fig. 3 ). On ROC analysis (Fig. 4), LA strain had the highest area under the curve (0.846). LA strain $<18.9 \%$ had a sensitivity of $62 \%$ and specificity of $95 \%$ for elevated PCWP, with likelihood ratio 12.3. LA strain trended with left ventricular ejection fraction $(r=0.33, P=0.07)$ and LA distensibility $(r=0.33, P=0.09)$, but did not reach statistical significance.

Measurement Reproducibility:

Tissue Doppler measurements had excellent reproducibility (Table III). LA distensibility also had good intra-observer and inter-observer reproducibility. LA strain measurements were less reproducible than LA volume or tissue Doppler measurements. 
TABLE III

Intra- and Inter-Observer Reproducibility

\begin{tabular}{|c|c|c|c|c|}
\hline & \multicolumn{2}{|c|}{ Intra-Observer } & \multicolumn{2}{|c|}{ Inter-Observer } \\
\hline & $\operatorname{cov}(\%)$ & ICC (95\% CI) & $\operatorname{cov}(\%)$ & ICC (95\% CI) \\
\hline E/E' ratio (lateral annulus) & 4.6 & $0.99(0.98-1.00)$ & 8.0 & $0.98(0.95-0.99)$ \\
\hline $\mathrm{E} / \mathrm{E}^{\prime}$ ratio (medial annulus) & 9.8 & $0.98(0.96-0.99)$ & 12.9 & $0.97(0.93-0.98)$ \\
\hline Velocity of propagation (Vp) & 18.7 & $0.89(0.77-0.95)$ & 29.7 & $0.71(0.46-0.88)$ \\
\hline E/Vp ratio & 13.2 & $0.95(0.89-0.98)$ & 30.4 & $0.67(0.41-0.86)$ \\
\hline Left atrial distensibility & 13.8 & $0.93(0.85-0.97)$ & 17.5 & $0.90(0.78-0.95)$ \\
\hline Left atrial strain & 18.4 & $0.89(0.77-0.95)$ & 25.9 & $0.75(0.54-0.88)$ \\
\hline
\end{tabular}

$\mathrm{Cl}=$ confidence interval; $\mathrm{COV}$ = coefficient of variability; $\mathrm{E}=$ peak early mitral inflow velocity; $\mathrm{E}^{\prime}$ = peak early mitral annular velocity; ICC = intra-class correlation.

\section{Discussion:}

Left atrial strain or distensibility predicts PCWP in pediatric heart transplant recipients better than traditional $E / E^{\prime}$ measurement. Of these two measures, LA strain best differentiates normal versus elevated PCWP, but is less reproducible than LA distensibility. To our knowledge, this is the first study to evaluate the relation of LA echocardiographic parameters and left ventricular filling pressure in this population.

\section{Limitations of Current Measurements:}

Left ventricular filling pressure is an important clinical measure in this population, as it may reflect subclinical changes due to rejection or transplant coronary artery disease. ${ }^{1,2}$ A noninvasive measure could allow more targeted invasive pressure measurement and earlier diagnosis and treatment. However, commonly used noninvasive surrogates, such as $E / E^{\prime}$ ratio, have important limitations. In an adult population, Ommen et al. ${ }^{4}$ found that mean LV diastolic pressure did not correlate well in patients with $E / E^{\prime}$ of 8-15, and correlated less well in patients with LVEF $>50 \%$. There may be particular limitations of $E /$ $E^{\prime}$ in the pediatric population. Border et al. ${ }^{7}$ demonstrated no significant correlation between $\mathrm{E} / \mathrm{E}^{\prime}$ and invasively measured LVEDP in pediatric patients with normal LV ejection fraction. In a pediatric heart transplant population, Eun et al. ${ }^{2}$ found only a weak correlation between $E / E^{\prime}$ and PCWP, which did not reach statistical significance. Our data support the limitations of this parameter within this population.

\section{Left Atrial Function:}

The left atrium serves multiple functions, with three distinct phases: a reservoir phase accepting pulmonary venous return during ventricular systole, a conduit phase for passive emptying with the mitral valve open in early diastole, and a contraction phase with active emptying at end-diastole. Left atrial function and left ventricular function are inter-related, as demonstrated by the correlation of left ventricular ejection fraction with LA distensibility, and the trend with LA strain. In the face of increased left ventricular diastolic dysfunction, the LA experiences an increased afterload, resulting in higher than normal LA pressure. This persistent increase in LA pressure leads to chamber enlargement, myocardial remodeling, decreased compliance, and impaired LA function. ${ }^{9,15,16}$ Due to the interdependence of the LA and left ventricle, and diastolic coupling, LA parameters reflect left ventricular filling pressure. Decreased atrial strain or distensibility denotes a less compliant left atrium, and thus a larger increase in pressure for a given increase in LA volume, which explains the inverse correlation with PCWP.

It is not surprising that neither maximum nor minimum LA volume related to PCWP in this population, as the left atrium status post heart transplant consists of both donor and recipient tissue, with absolute LA volume varying by technique. Changes in left atrial volume or deformation, as described by distensibility and strain, are a manifestation of the interplay of left atrial and left ventricular function. However, although LA distensibility and strain may better reflect LV filling pressure than $E / E^{\prime}$ in this population, the correlation with PCWP is still modest, compared with stronger reported correlation in adults with normal anatomy. ${ }^{9}$ This may reflect the variability in a hybrid left atrium following heart transplant. However, with current techniques of bicaval anastomosis, the majority of atrial tissue is donor atrium.

Decreased LA strain better predicts elevated PCWP than decreased LA distensibility, with higher area under the curve on ROC analysis. However, this measure may have clinical limitations, as its analysis requires specialized software and/or analysis of proprietary data, and is less reproducible than distensibility. The lower 
reproducibility of LA strain may reflect difficulty in tracking the thin atrial wall. The abnormal geometry of the left atrium and anastomotic line may further negatively affect tracking algorithms. Further refinement is necessary to improve techniques and limit variability.

\section{Limitations:}

The small sample size limits the ability for subgroup analysis, particularly with the small number of patients with rejection or coronary vasculopathy. Comparisons were made to PCWP rather than LVEDP due to sample size limitations, however, PCWP is a commonly used measure, and the close correlation of PCWP and LVEDP suggests this is an appropriate surrogate. This study assumes similar hemodynamic and loading conditions at the time of catheterization and echocardiogram. Although the level of sedation may not be identical between these studies, this assumption is likely reasonable given the close temporal relationship. In addition, screening echocardiograms would not be performed with sedation, and we feel that this better reflects real clinical use. LA strain was measured in only one plane, which may not fully characterize global deformation, but still appears to reflect left atrial function, both from the current data and prior report. ${ }^{10}$ Due to the cross-sectional nature of this study, we were unable to determine which measures best track changes in hemodynamics over time, or which measures predict clinical endpoints such as rejection or TCAD. Although this cohort demonstrated a range of PCWP typical for this population, extremes of PCWP were not well represented in this small sample size.

\section{Conclusions:}

LA reservoir function is a more useful surrogate of left ventricular filling pressure than $E / E^{\prime}$ in the pediatric heart transplant population. Peak LA systolic strain best differentiates normal versus elevated PCWP, but may be limited by measurement variability. Further study is necessary to improve reproducibility and to evaluate longitudinal changes in pressure and clinical outcomes.

\section{References}

1. Aiyagari R, Nika M, Gurney JG, et al: Association of pediatric heart transplant coronary vasculopathy with abnormal hemodynamic measures. Congenit Heart Dis 2011;6:128-133.

2. Eun LY, Gajarski RJ, Graziano JN, et al: Relation of left ventricular diastolic function as measured by echocardiography and pulmonary capillary wedge pressure to rejection in young patients ( $<$ or $=31$ years) after heart transplantation. Am / Cardiol 2005;96:857-860.

3. Nagueh SF, Middleton KJ, Kopelen HA, et al: Doppler tissue imaging: a noninvasive technique for evaluation of left ventricular relaxation and estimation of filling pressures. J Am Coll Cardiol 1997;30:1527-1533.

4. Ommen SR, Nishimura RA, Appleton CP, et al: Clinical utility of Doppler echocardiography and tissue Doppler imaging in the estimation of left ventricular filling pressures: A comparative simultaneous Doppler-catheterization study. Circulation 2000;102:1788-1794.

5. Dokainish H, Zoghbi WA, Lakkis NM, et al: Optimal noninvasive assessment of left ventricular filling pressures: a comparison of tissue Doppler echocardiography and Btype natriuretic peptide in patients with pulmonary artery catheters. Circulation 2004;109:2432-2439.

6. Rivas-Gotz C, Manolios M, Thohan V, et al: Impact of left ventricular ejection fraction on estimation of left ventricular filling pressures using tissue Doppler and flow propagation velocity. Am J Cardiol 2003;91:780-784.

7. Border WL, Michelfelder EC, Glascock B], et al: Color Mmode and Doppler tissue evaluation of diastolic function in children: simultaneous correlation with invasive indices. J Am Soc Echocardiogr 2003;16:988-994.

8. Bhella PS, Pacini EL, Prasad A, et al: Echocardiographic indices do not reliably track changes in left-sided filling pressure in healthy subjects or patients with heart failure with preserved ejection fraction. Circ Cardiovasc Imaging 2011;4:482-489.

9. Hsiao SH, Lin KL, Chiou KR: Comparison of left atrial volume parameters in detecting left ventricular diastolic dysfunction versus tissue Doppler recordings. Am / Cardiol 2012; 109:748-755.

10. Kurt M, Tanboga IH, Aksakal E, et al: Relation of left ventricular end-diastolic pressure and $\mathrm{N}$-terminal pro-brain natriuretic peptide level with left atrial deformation parameters. Eur Heart J Cardiovasc Imaging 2012;13:524530.

11. Wakami K, Ohte N, Asada K, et al: Correlation between left ventricular end-diastolic pressure and peak left atrial wall strain during left ventricular systole. / Am Soc Echocardiogr 2009;22:847-851.

12. Liu $Y$, Wang $K$, Su D, et al: Noninvasive assessment of left atrial phasic function in patients with hypertension and diabetes using two-dimensional speckle tracking and volumetric parameters. Echocardiography 2014;31:727735.

13. Kim DG, Lee KJ, Lee $S$, et al: Feasibility of two-dimensional global longitudinal strain and strain rate imaging for the assessment of left atrial function: a study in subjects with a low probability of cardiovascular disease and normal exercise capacity. Echocardiography 2009;26: 1179-1187.

14. Nagueh SF, Appleton CP, Gillebert TC, et al: Recommendations for the evaluation of left ventricular diastolic function by echocardiography. I Am Soc Echocardiogr 2009; 22:107-133.

15. Abhayaratna WP, Seward JB, Appleton CP, et al: Left atrial size: physiologic determinants and clinical applications. J Am Coll Cardiol 2006;47:2357-2363.

16. Otani K, Takeuchi M, Kaku K, et al: Impact of diastolic dysfunction grade on left atrial mechanics assessed by two-dimensional speckle tracking echocardiography. J Am Soc Echocardiogr 2010;23:961-967. 